\title{
Digestibilidade de dietas contendo antibiótico, probiótico e prebiótico para coelhos em crescimento
}

\author{
Joseli Alves Ferreira Zanato ${ }^{1}$ \\ Jeffrey Frederico Lui ${ }^{{ }^{*}}$ \\ Maria Cristina Oliveira ${ }^{2}$ \\ Aderbal Cavalcante Neto ${ }^{1,3}$ \\ Otto Mack Junqueira ${ }^{1}$ \\ Euclides Braga Malheiros ${ }^{1}$ \\ Claúdio Scapinello ${ }^{1,4}$ \\ ${ }^{1} \mathrm{PPG}$ em Zootecnia, FCAV/UNESP \\ Via de Acesso Prof. Paulo Donato Catellane, s/n, CEP 14884-900, Jaboticabal-SP, Brasil \\ ${ }^{2}$ Universidade de Rio Verde, Fazenda Fontes do Saber \\ Campus Universitário, s/n, CEP 75901-910, Rio Verde-GO, Brasil \\ ${ }^{3}$ Universidade de Aveiro, CESAM, Portugal \\ ${ }^{4}$ Universidade Estadual de Maringá, \\ Av. Colombo, 5790, CEP 87020-900, Maringá-PR, Brasil \\ *Autor para contato \\ jeffrey@fcav.unesp.br
}

Submetido em 22/04/2008

Aceito para publicação em 07/07/2008

\section{Resumo}

Objetivou-se avaliar a utilização de antibiótico, probiótico e prebiótico sobre a digestibilidade de nutrientes de dietas para coelhos em crescimento. Utilizaram-se 20 coelhos, com 50 dias de idade. O delineamento foi o inteiramente casualizado, com cinco tratamentos e quatro repetições. Os tratamentos (T) utilizados foram os seguintes: $\mathrm{T}_{1}=$ ração controle negativo (sem aditivo promotor de crescimento); $\mathrm{T}_{2}=$ ração com antibiótico, controle positivo ( $0,05 \%$ de bacitracina de zinco); $\mathrm{T}_{3}=$ ração com probiótico $(0,15 \%$ de Bacillus subtillis na concentração de $\left.10^{9} \mathrm{UFC} / \mathrm{g}\right) ; \mathrm{T}_{4}=$ ração com prebiótico ( $0,15 \%$ de mananoligossacarídeo fosforilado a $\left.30 \%\right)$; e $\mathrm{T}_{5}=$ ração com simbiótico (probiótico + prebiótico). O período experimental teve duração de 12 dias, sendo sete de adaptação às dietas experimentais e às gaiolas e cinco dias de coleta. Foram calculados os coeficientes de digestibilidade aparente da matéria seca, matéria orgânica, proteína bruta, fibra em detergente neutro, fibra em detergente ácido, matéria mineral e extrato etéreo. Só foi observada influência significativa do tratamento sobre os coeficientes de fibra em detergente ácido e da matéria seca, nos quais o prebiótico apresentou maiores valores. Conclui-se que a adição de probiótico e simbiótico à ração resultou em coeficientes de digestibilidade dos coelhos semelhantes aos que receberam as dietas com antibióticos e sem nenhum aditivo. Melhores resultados de digestibilidade foram obtidos com a utilização do prebiótico.

Unitermos: aditivos, bacillus subtillis, mananoligossacarídeo, nutrição animal 


\section{Abstract}

Digestibility of diets containing an antibiotic, a probiotic and a prebiotic in growing rabbits. This study aimed to evaluate the effects of a probiotic and/or prebiotic additive on the nutrient digestibility of diets for growing rabbits. Twenty 50-day-old rabbits were used. A completely randomized experimental design, with five treatments and four replicates each, was used. The applied treatments $(\mathrm{T})$ were the following: $\mathrm{T}_{1}=$ negative control diet (no growth promoter); $\mathrm{T}_{2}=$ diet with antibiotic, positive control ( $0.05 \%$ zinc bacitracin); $\mathrm{T}_{3}=$ diet with probiotic $\left(0.15 \%\right.$ Bacillus subtillis at a concentration of $\left.10^{9} \mathrm{UFC} / \mathrm{g}\right) ; \mathrm{T}_{4}=$ diet with prebiotic $(0.15 \%$ phosphorylated mananoligossaccharide at $30 \%)$; and $\mathrm{T}_{5}=$ diet with symbiotic (probiotic + prebiotic). The experimental period lasted 12 days, seven for adaptation to the experimental diets and to the cages, and five days of feces collection. The apparent digestibility coefficients of dry matter, organic matter, crude protein, neutral detergent fiber, acid detergent fiber, mineral matter, and ether extract were calculated. The addition of a probiotic and of a symbiotic to the rabbit diet resulted in digestibility similar to that of animals fed diets containing antibiotics or no additive at all. The best digestibility results were obtained with the prebiotic.

Key words: additives, Bacillus subtillis, mannanoligosaccharide, animal nutrition

\section{Introdução}

No processo de digestão dos alimentos, apenas uma parte é metabolizada e retida na forma de tecidos estruturais de reserva ou, ainda, transformada em produtos para secreção exógena, endógena ou mista. A outra parte é perdida na forma de gases ou calor resultante do metabolismo. Segundo Santos et al. (2004), o estudo da eficiência da utilização dos nutrientes de um alimento inicia-se com o conhecimento da sua composição química e do seu valor calórico, seguindo com a análise dos coeficientes de digestibilidade dos nutrientes e do desempenho do animal. Esses coeficientes podem ser influenciados pelo tipo de alimento, se volumoso ou concentrado (Carabaño et al., 2001), pela espécie ou variedade, quando forrageira (Pérez et al., 1995), e pela forma física apresentada pelo alimento, se grosseira ou finamente moído (Gomes et al., 2000).

A ação positiva dos antibióticos deve-se à sua influência sobre a biota intestinal, controlando as infecções subclínicas e potencializando a absorção de nutrientes (De Blas, 1984). Sendo assim, as características esperadas de um produto alternativo aos antibióticos devem ser a capacidade de: alterar o pH intestinal; manter mucinas protetoras do intestino; selecionar organismos benéficos ou atuar contra patógenos; e aumentar a fermentação ácida, a absorção de nutrientes e a resposta imune humoral.

Segundo Fuller (1989), os probióticos são suplementos alimentares à base de microrganismos vivos que afetam beneficamente o animal hospedeiro, promovendo o balanço da biota intestinal. Seu objetivo é criar uma barreira intestinal contra agentes patógenos e alguns deles têm se mostrado benéficos aos desempenhos dos coelhos (De Blas et al., 1991). Outros, entretanto, não exercem efeito (Maertens et al., 1994). Já os prebióticos são considerados ingredientes não digestíveis que estimulam o crescimento e/ou a atividade de um limitado número de microorganismos capazes de proporcionar um ambiente intestinal saudável ao hospedeiro (Gibson e Roberfroid, 1995).

O uso dos probióticos pode melhorar a digestibilidade de diversos nutrientes. De acordo com Yamani et al. (1992), a suplementação de dietas com o probiótico Lacto-Sacc melhorou a digestibilidade da fibra bruta. Mais recentemente, vários autores relataram maiores coeficientes de digestibilidade da matéria seca e da orgânica (Zoccarato et al., 1995) e proteína bruta (Kamra et al., 1996), com dietas suplementadas com Bacillus subtilis + B. licheniformes (Kermauner e Struklec, 1996), e verificaram uma melhor digestibilidade da fibra bruta, do extrato etéreo e da matéria mineral com o uso de probióticos compostos por Bacillus sp.. Contudo Hollister et al. (1989) e Michelan et al. (2002) não observaram diferenças nos coeficientes de digestibilidade aparente da matéria seca, matéria orgânica, proteína bruta, fibra em detergente neutro e energia bruta, devido ao uso de probióticos, ácidos orgânicos e antibióticos. Logo se objetivou avaliar a inclusão de antibiótico, probiótico e prebiótico sobre a digestibilidade de nutrientes de dietas para coelhos em crescimento. 


\section{Material e Métodos}

Os ensaios com os animais foram conduzidos no Setor de Cunicultura do Departamento de Zootecnia da Universidade Estadual Paulista, campus de Jaboticabal. Foram utilizados 20 coelhos da raça Nova Zelândia Branca, com 50 dias de idade, alojados individualmente em gaiolas de metabolismo, providas de bebedouro automático, comedouro semi-automático e dispositivo para coleta de fezes. $\mathrm{O}$ delineamento experimental foi inteiramente casualizado, com cinco tratamentos e quatro repetições. A unidade experimental foi constituída por um animal.

Os tratamentos $(\mathrm{T})$ utilizados foram os seguintes: $\mathrm{T}_{1}=$ ração controle negativo (sem aditivo promotor de crescimento); $\mathrm{T}_{2}=$ ração com antibiótico, controle positivo $\left(0,05 \%\right.$ de bacitracina de zinco); $\mathrm{T}_{3}=$ ração com probiótico $(0,15 \%$ de Bacillus subtillis na concentração de $\left.10^{9} \mathrm{UFC} / \mathrm{g}\right) ; \mathrm{T}_{4}=$ ração com prebiótico $(0,15 \%$ de mananoligossacarídeo fosforilado a $30 \%$ ); e $\mathrm{T}_{5}=$ ração com simbiótico (probiótico + prebiótico).

O probiótico utilizado, Biotop ${ }^{\circledR}$, era composto de células vivas e viáveis de Bacillus subtillis, na concentração de $10^{9}$ U.F.C./g. O prebiótico utilizado era composto de mananoligossacarídeos e foi adicionado à ração de acordo com as instruções do fabricante (Fatec). O antibiótico utilizado foi a bacitracina de zinco (12\%).

O suplemento vitamínico-mineral utilizado era desprovido de promotor de crescimento, coccidiostático ou antibiótico, com o objetivo de evitar interferências destes produtos nos processos digestivos do animal.

A composição percentual e calculada da ração peletizada (Tabela 1) foi a mesma para todos os

TABELA 1: Composição percentual e química das dietas experimentais.

\begin{tabular}{|c|c|c|c|c|c|}
\hline \multirow{2}{*}{ Ingredientes (kg) } & \multicolumn{5}{|c|}{ Tratamentos } \\
\hline & Sem aditivo & Antibiótico & Probiótico & Prebiótico & Simbiótico \\
\hline Milho & 25,64 & 25,64 & 25,64 & 25,64 & 25,64 \\
\hline Farelo de soja & 9,80 & 9,80 & 9,80 & 9,80 & 9,80 \\
\hline Farelo de trigo & 14,71 & 14,71 & 14,71 & 14,71 & 14,71 \\
\hline Feno de alfafa & 39,32 & 39,32 & 39,32 & 39,32 & 39,32 \\
\hline Casca de arroz & 6,08 & 6,08 & 6,08 & 6,08 & 6,08 \\
\hline Óleo de soja & 0,98 & 0,98 & 0,98 & 0,98 & 0,98 \\
\hline Sal & 0,49 & 0,49 & 0,49 & 0,49 & 0,49 \\
\hline Fosfato bicálcico & 1,18 & 1,18 & 1,18 & 1,18 & 1,18 \\
\hline DL-metionina 99\% & 0,20 & 0,20 & 0,20 & 0,20 & 0,20 \\
\hline Suplemento de vitaminas + minerais* & 0,39 & 0,39 & 0,39 & 0,39 & 0,39 \\
\hline Lignossulfonato de sódio & 0,98 & 0,98 & 0,98 & 0,98 & 0,98 \\
\hline Caulim & 0,35 & 0,30 & 0,20 & 0,20 & 0,05 \\
\hline Antibiótico** & - & 0,05 & - & - & - \\
\hline Probiótico*** & - & - & 0,15 & - & 0,15 \\
\hline Prebiótico*** & - & - & - & 0,15 & 0,15 \\
\hline \multirow[t]{2}{*}{ Total } & 100,00 & 100,00 & 100,00 & 100,00 & 100,00 \\
\hline & \multicolumn{5}{|c|}{ Composição química $^{1}$} \\
\hline Matéria seca $(\%)$ & 90,22 & 90,22 & 90,22 & 90,22 & 90,22 \\
\hline Proteína bruta (\%) & 17,50 & 17,50 & 17,50 & 17,50 & 17,50 \\
\hline Energiadigestível $^{2}$ (kcal/kg) & 2500 & 2500 & 2500 & 2500 & 2500 \\
\hline Fibra bruta $(\%)$ & 14,94 & 14,94 & 14,94 & 14,94 & 14,94 \\
\hline FDA $(\%)$ & 20,21 & 20,21 & 20,21 & 20,21 & 20,21 \\
\hline FDN (\%) & 34,74 & 34,74 & 34,74 & 34,74 & 34,74 \\
\hline
\end{tabular}

FDN: fibra em detergente neutro; FDA: fibra em detergente ácido; *por quilograma de ração na matéria natural - composição por kg do produto: Vit A, 1.875.000 UI; Vit D3, 250.000 UI: Vit E, 12.500 mg; Vit K3, 750 mg; Vit B1, 500 mg; Vit B2, 1.000 mg; Vit B6, 600 mg; Vit B12, 2.500 mcg; Ácido Pantotênico, 2000 mg; Colina, 125 g; Fe, 80.000 mg; Cu, 12.000 mg; Mn, 60.000 mg; Zn, 50.000 mg; I, 5.000 mg; Se, 15 mg; Antioxidante, 25g.; ** bacitracina de zinco (12\%). ***Bacillus subtillis, na concentração de $10^{9}$ U.F.C./g; ***mananoligossacarídeo fosforilado a 30\%; ${ }^{1}$ Análises realizadas no laboratório de Nutrição Animal do Departamento de Zootecnia - Unesp - Jaboticabal; ${ }^{2}$ Com base nos valores de composição químicos das matérias-primas das rações. 
tratamentos, variando apenas o aditivo. A ração foi formulada de acordo com as exigências nutricionais para coelhos em crescimento (Lebas, 1989) e sua composição foi calculada com base em Rostagno et al. (2000).

O período experimental teve duração de 12 dias, sendo sete de adaptação às dietas experimentais e às gaiolas e cinco dias de coleta de fezes. As rações e a água eram fornecidas à vontade durante a primeira fase do experimento, para que o consumo voluntário fosse estimado. Na segunda fase, o animal recebeu $90 \%$ do seu consumo voluntário, para que não houvesse sobras de ração.

As fezes de cada animal foram coletadas em sua totalidade uma vez ao dia, no período da manhã, acondicionadas em sacos plásticos e armazenadas em congelador a $-18^{\circ} \mathrm{C}$. Posteriormente, ao final do experimento, essas fezes foram homogeneizadas e secas em estufa de ventilação forçada a $55^{\circ} \mathrm{C}$, durante $72 \mathrm{~h}$.

Nas análises químicas das rações e das fezes, determinaram-se os teores de matéria seca (MS), proteína bruta (PB), extrato etéreo (EE), matéria mineral (MM), fibra em detergente neutro (FDN) e fibra em detergente ácido (FDA), conforme metodologias descritas por Silva e Queiroz (2002).

Em seguida, foram calculados os coeficientes de digestibilidade aparente da matéria seca (CDMS), matéria orgânica (CDMO), proteína bruta (CDPB), fibra em detergente neutro (CDFDN), fibra em detergente ácido (CDFDA), matéria mineral (CDMM) e extrato etéreo (CDEE).

Os dados foram analisados, utilizando-se o SAS (1999) e, em caso de significância estatística, as médias foram submetidas ao teste de Tukey (5\%). O modelo utilizado pode ser representado como segue: $\mathrm{Y}_{\mathrm{ij}}=\mu+\mathrm{A}_{\mathrm{i}}+\mathrm{e}_{\mathrm{ij}}$

Em que: $\mathrm{Y}_{\mathrm{ij}}=$ parâmetro avaliado; $\mu=$ média geral do parâmetro; $\mathrm{A}_{\mathrm{i}}=$ efeito do i-ésimo aditivo na dieta; e $\mathrm{e}_{\mathrm{ij}}=$ erro aleatório associado a cada observação, com $\mu=0$ e $\delta_{e}^{2}$.

\section{Resultados e Discussão}

Verifica-se, na tabela 2, que houve diferença significativa $(\mathrm{P}<0,01)$ para o coeficiente de digestibilidade da fibra em detergente ácido (CDFDA), na qual se observou que os coelhos que receberam ração com prebiótico diferiram dos demais, apresentando maior digestibilidade. Ainda analisando a tabela 2, nota-se que também houve significância $(\mathrm{P}<0,01)$ para o coeficiente de digestibilidade da matéria seca, sendo também o tratamento com prebiótico o que apresentou estatisticamente média superior aos demais.

Embora vários autores (De Blas, 1984; Fuller, 1989; Kermauner e Strucklec, 1996) afirmem que a utilização de aditivos em dietas, como probiótico e antibióticos, atuam controlando a proliferação de microrganismos patógenos, favorecendo a proliferação de microrganismos desejáveis e/ou atuando sobre o $\mathrm{pH}$ do trato digestivo e, como resultado, diminui os distúrbios gastrointestinais e melhora o aproveitamento dos nutrientes e o desempenho dos animais, pôde-se verificar que as análises dos coeficientes de digestibilidade não foram, na totalidade, significativos como se esperava entre os tratamentos.

Notou-se que, para alguns parâmetros, o prebiótico apresentou efeito significativo em relação aos demais tratamentos. Associado a isso, Spring (2000) sugeriu que os prebióticos podem atuar bloqueando os sítios de ligação de bactérias patogênicas na mucosa intestinal, diminuindo, assim, os danos à mucosa e, conseqüentemente, o “turnover" (síntese, migração e extrusão) dessas células, o que pode resultar em melhor utilização dos ingredientes da dieta, uma vez que ocorre a manutenção do tamanho dos vilos e, portanto, a manutenção da capacidade digestiva e de absorção intestinal. 
TABELA 2: Coeficientes de digestibilidade para proteína bruta (CDPB), fibra em detergente ácido (CDFDA), fibra em detergente neutro (CDFDN), extrato etéreo (CDEE), matéria seca (CDMS), matéria mineral (CDMM) e matéria orgânica (CDMO).

\begin{tabular}{|c|c|c|c|c|c|c|}
\hline \multirow[b]{2}{*}{ Coeficiente } & \multicolumn{5}{|c|}{ Tratamentos } & \multirow[b]{2}{*}{ CV (\%) } \\
\hline & $\begin{array}{c}1 \\
\text { (controle) }\end{array}$ & $\begin{array}{c}2 \\
\text { (antibiótico) }\end{array}$ & $\begin{array}{c}3 \\
\text { (probiótico) }\end{array}$ & $\begin{array}{c}4 \\
\text { (prebiótico) }\end{array}$ & $\begin{array}{c}5 \\
\text { (simbiótico) }\end{array}$ & \\
\hline CDPB & 77,89 & 76,07 & 76,38 & 81,40 & 73,67 & 5,18 \\
\hline CDFDA & $26,62^{\mathrm{bc}}$ & $27,72^{\mathrm{b}}$ & $20,10^{c}$ & $45,3^{\mathrm{a}}$ & $26,88^{\mathrm{bc}}$ & 10,94 \\
\hline CDFDN & 41,38 & 33,64 & 31,42 & 49,53 & 30,10 & 25,21 \\
\hline CDEE & 94,60 & 91,01 & 92,91 & 93,81 & 89,72 & 3,85 \\
\hline CDMS & $82,27 b$ & $80,72 b$ & $81,25 b$ & $88,40 \mathrm{a}$ & $80,86 b$ & 3,38 \\
\hline CDMM & 61,98 & 54,97 & 59,15 & 64,70 & 55,56 & 10,68 \\
\hline CDMO & 64,66 & 62,50 & 61,38 & 67,96 & 60,16 & 9,44 \\
\hline
\end{tabular}

Médias, na mesma linha, seguidas de letras desiguais diferem pelo teste de Tukey $(\mathrm{p}>0,05)$.

Embora os mananoligossacarídeos (MOS) sejam usados como prebióticos, eles não são seletivamente benéficos para um grupo específico de bactérias, ao invés disso, eles agem estimulando a proliferação de bactérias que produzem ácidos, como os lactobacilos e bifidobactérias, portanto, sua inclusão em dietas para coelhos pode melhorar a saúde do animal, a digestibilidade de nutrientes e, conseqüentemente, o desempenho produtivo.

De acordo como a literatura, o uso dos probióticos pode melhorar a digestibilidade de diversos nutrientes, uma vez que Yamani et al. (1992) verificaram que a suplementação de dietas com o probiótico Lacto-Sacc melhorou a digestibilidade da fibra bruta, contrariando o encontrado nesse trabalho. Outros autores ainda relataram maiores coeficientes de digestibilidade da matéria seca, matéria orgânica (Zoccarato et al., 1995) e proteína bruta (Kamra et al., 1996), com dietas suplementadas com Bacillus subtillis $+B$. licheniformes. Kermauner e Struklec (1996) verificaram uma melhor digestibilidade da fibra bruta, do extrato etéreo e da matéria mineral com o uso de probióticos compostos por Bacillus sp.

Todavia, corroborando os resultados aqui obtidos, Hollister et al. (1989) e Michelan et al. (2002) não observaram diferenças nos coeficientes de digestibilidade aparente da matéria seca, matéria orgânica, proteína bruta, fibra em detergente neutro e energia bruta quando usaram probióticos, ácidos orgânicos e antibióticos.

Esses diferentes resultados encontrados na literatura podem estar associados à composição dos probióticos, à dosagem, às condições ambientais, à fase fisiológica dos animais, às características das dietas e, principalmente, ao nível de desafio sanitário do local. Nesse sentido, a ação dos probióticos depende, basicamente, da composição do produto e, segundo Fuller (1989), das características e sua relação com os microrganismos presentes no trato digestivo das diferentes espécies. Já Tournut (1998) ponderou que os efeitos benéficos de probióticos podem aparecer a partir de um determinado tempo, necessário para que o agente se estabeleça no trato digestivo e equilibre a flora.

A adição de probiótico e simbiótico à ração resultou em coeficientes de digestibilidade dos coelhos semelhantes aos que receberam as dietas com antibióticos e sem nenhum aditivo. Melhores resultados de digestibilidade foram obtidos com a utilização de prebiótico.

\section{Agradecimento}

Os autores agradecem a Fundação de Amparo à Pesquisa do Estado de São Paulo - FAPESP pelo financiamento total desta pesquisa. 


\section{Referências}

Carabaño, R.; García, J.; De Blas, J. C. 2001. Effect of fibre source on ileal apparent digestibility of non-starch polysaccharides in rabbits. Animal Science, 72: 343-350.

De Blas, C. 1984. Alimentación del conejo. Mundi-Prensa, Madrid, España, 215pp.

De Blas, C.; Garcia, J.; Alday, S. 1991. Effects of dietary inclusion of a probiotic (Paciflor) on performance of growing rabbits. Journal of Applied Rabbit Research, 14: 148-150.

Fuller, R. 1989. Probioticas in man and animals. A review. Journal of Applied Bacteriology, 66: 365-378.

Gibson, G. R.; Rorberfroid, M. B. 1995. Dietary modulation of the human colonic microbiota: Introducing the concept of prebiotics. Journal of Nutrition, 125: 1401-1412.

Gomes, A. V. C.; Rocha, J. C. C. ; Vieira, A. A.; Crespi, M. P. A. L. 2000. Effect of particle size of coast cross hay (Cynodon dactylon) on performance and diet digestibility in growing rabbits. Proceedings of the VII World Rabbit Congress, Valencia, España, p.249254.

Hollister, A. G.; Cheeke, P. R.; Robinson, K. L. 1989. Effects of water-administered probiotics and acidifiers on growth, feed conversion and enteritis mortality of weaning rabbits. Journal of Applied Rabbit Research, 12: 143-147.

Kamra, D. N.; Chaudhary, L. C.; Singh, R.; Pathak, N. N. 1996. Influence of feeding probiotics on growth performance and nutrient digestibility in rabbits. World Rabbit Science, 4: 85-88.

Kermauner, A.; Struklec, M. 1996. Addition of probiotic to feeds with different energy and ADF content in rabbits. 1. Effect on digestive organs. World Rabbit Science, 4: 187-193.

Lebas, F. 1989. Besoins nutritionnels des lapins: Revue bibliographique et perspectives. Cuniculture Science, 5: 1-28.

Maertens, L.; Van Renterghem, R.; De Groote, G. 1994. Effects of dietary inclusion of Paciflor (Bacillus CIP 5832) on the milk composition and performance of does on caecal and growth parameters of their weanlings. World Rabbit Science, 2: 67-73.
Michelan, A. C.; Scapinello, C.; Natal, M. R. M.; Furlan, A. C.; Sakaguti, E. S.; Faria, H. G.; Santolin, M. L. R.; Hernandes, A. B. 2002. Utilização de probiótico, ácido orgânico e antibiótico em dietas para coelhos em crescimento: ensaio de digestibilidade, avaliação da morfometria intestinal e desempenho. Revista Brasileira de Zootecnia, 31: 2227-2237.

Pérez, J. M.; Lebas, F.; Gidenne, T. 1995. European reference method for in vivo determination of diet digestibility in rabbits. World Rabbit Science, 3: 41-43.

Rostagno, H. S.; Albino, L. F. T.; Donzele, J. L.; Gomes, P. C.; Ferreira, A. S.; Oliveira, R. F. M.; Lopes, D. C. 2000. Tabelas brasileiras para aves e suínos: Composição de alimentos e exigências nutricionais. UFV, Viçosa, Brasil, 141pp.

Santos, E. A.; Lui, J. F.; Scapinello, C. 2004. Efeito dos níveis de fibra em detergente ácido sobre os coeficientes de digestibilidade das dietas e desempenho de coelhos em crescimento. Acta Scientiarun. Animal Sciences, 26: 79-86.

Spring, P. 2000. Yeast's secret weapon aids animal production. Anais do Simpósio sobre Aditivos Alternativos na Nutrição Animal, Campinas, Brasil, p.41-50.

SAS - Statistical Analysis System Institute. 1999. SAS User's Guide: Statistics version 6. $4^{\text {th }}$ ed. SAS Institute Inc., Cary, USA, 1686pp.

Silva, D. J.; Queiroz, C. A. 2002. Análise de alimentos: Métodos químicos e biológicos. $3^{\mathrm{a}}$ ed. UFV, Viçosa, Brasil, 235pp.

Tournut, J. R. 1998. Probiotics. Anais da XXXV ${ }^{\text {a }}$ Reunião Anual da Sociedade Brasileira de Zootecnia, Botucatu, Brasil, p.179199.

Yamani, K. A.; Ibrahim, H.; Rashwan, A. A. 1992. Effects of a pelleted diet supplemented with probiotic (Lacto-Sacc) and water supplemented with a combination of probiotic and acidifier (Acid Pak-4-Way) on digestibility, growth, carcass and physiological aspects of weanling New Zealand White rabbits. Journal of Applied Rabbit Research, 15: 1087-1100.

Zoccarato, I.; Barbera, S.; Tartari, E. 1995. Effetto dell'impiego di mangine contenente um'associazione antibiotico-probiotico sulle performance del coniglio all'ingrasso. Zootecnica e Nutrizione Animal, 21: 297-304. 